\title{
RECONSTRUCTING THE DATABASE AFTER ELECTRONIC ATTACKS
}

Brajendra Panda and Joseph Giordano

\begin{abstract}
In spite of all existing security mechanisms, it is quite difficult to protect databases from electronic attacks. This research provides techniques to make an assessment of the damaged data and then to recover the affected data to consistent states after an attack is detected. Damage assessment is done using data dependency approach in order to obtain precise information on the damaged part of the database. Two algorithms are presented in this paper. The first algorithm performs the damage assessment and recovery simultaneously; whereas the second algorithm separates these two processes for improved efficiency. Both algorithms allow blind- writes on data items allowing damaged items to be recovered automatically.
\end{abstract}

\subsection{INTRODUCTION}

With the increasing popularity of Internet, worldwide information sharing becomes a common practice. At the same time, this connectivity with the rest of the world opens channels for intruders to access and possibly damage sensitive information. Although there are several techniques available, as described in [1] and [4], to prevent unauthorized access to sensitive data, these preventive measures are not always successful. It seems extremely hard to build systems that share information over electronic networks and still remain invulnerable to attackers. Hackers are always in search of new ways to prevail over the system security. Password sniffing and session hijackings are among various other means of intruding into a system, and the system will not be able to detect an attacker from a legitimate user in these cases. Besides, there remains possibility of significant damage by insider-turn-foes. 
The productivity of any organization heavily depends on the information it shares with and protects from the rest of the world. An attack on an organization's information resources can have significantly devastating impact on the ability of the organization. Such an attack through electronic media is called Information Warfare. Defensive information warfare consists of three major phases: prevention, detection, and recovery from attacks. Various preventive measures to protect the databases from intruders in a defensive information warfare environment have been discussed in [1] and [4]. There are several ways to detect an intrusion into a system. Of these, a statistical approach has been discussed in [7] and a knowledge-based approach has been offered in [9]. Storage jamming [10] and [11], a method for misleading attackers to access fake data, can also be used to detect the intrusion. A more detailed discussion of intrusion detection techniques can be found in [8]. Pattern matching against known process of attack, examination of statistical profiles, inspection of known values of data, for example, are few others of these methods. Sometimes an attack may go unnoticed for a while, and as a result, the damaged data may spread and corrupt other undamaged data through other users. For example, a legitimate user may use the value of a corrupt data and update several other uncorrupted data based on the value read. This can have a cascading effect over time. Therefore, it is of utmost importance that the database be reconstructed by repairing the damage as soon as an attack is detected.

Traditional recovery methods [2], [3], [5], [6] fail to provide the integrity and efficacy needed to react to the situation under consideration. These issues are discussed in the next section. The objective of this research is to make an exact assessment of the damaged data when an attack is detected and then recover the affected data to a consistent state. As hindering the activities of other users of the system may be the intention of the attacker, it is desirable that the recovery process must bring the system back in real-time, while maintaining system integrity to the maximum extent possible.

In section 2, we examine various recovery methods and their shortcomings in defensive information warfare environment. Section 3 introduces our recovery model. A graph-based approach to the damage assessment is introduced in section 4. The algorithms are presented in section 5 . Section 6 offers the conclusion of this research.

\subsection{MOTIVATION}

Conventional recovery algorithms presented in [2], [3], [5], [6] use a log to register each write operation of a transaction. During a system failure, the effects of all write operations of non-committed transactions that are already written into the stable database are undone. Furthermore, the effects of all write operations of committed transactions are redone if they are not in the stable database. This guarantees the integrity and consistency of the database. The $\log$ is also temporarily purged when it is determined that the stable database reflects the updates of all committed transactions (thus requiring no redo) and no effects of any of the non-committed transactions (thus requiring no undo). 
The recovery method does not require any read operations for any of the transactions. The transactions are also never stored entirely since only the before images and after images are required during the undo and redo processes.

The above approach does not work when there is a malicious transaction that has already updated few data items and has committed. The system treats the attacker as any other valid transaction and makes the update permanent. This is guaranteed by the ACID properties (Atomicity, Consistency preservation, Isolation, and Durability) of transactions. Whenever the attacker is detected, all the updates of the attacker must be undone including the updates of the transactions that directly or transitively read from the attacker. Then, these valid transactions must be re-executed to return the database to a consistent state. However, this is not possible for the following two reasons. First, as the $\log$ does not store the read operations, the read-from relationships can not be determined. Secondly, since the transactions are never stored entirely, redoing the valid transactions is impossible. Therefore, it is necessary to update the $\log$ to store all operations of each transaction in the log. Nevertheless, it is not efficient to re-execute all transactions from the point of attack. For example, if an attack is detected after a month of its occurrence, it requires significant amount of time to undo and then redo all the transactions that have directly or indirectly read-from the attacking transaction. Besides, the system remains unavailable to users during the recovery process and thus yields to denial of service. In most military and some commercial applications, denial of service is highly undesirable. Therefore, development of an efficient algorithm to recover the system from electronic attacks is quite essential.

\subsection{RECOVERY MODEL}

As stated earlier, we assume that the database has been attacked and the attacking transaction has been detected. The method of detection is beyond the scope of this paper. This research is based on the following additional assumptions: (1) the scheduler produces a strict serializable history, (2) the log stores all operations of each transaction, and the order of operations in the log is the same as that in the history, and (3) the log is not modifiable by users (so that an attacker will not be able to damage the log).

Definition 17 A write operation $w_{i}[x]$ of a transaction $T_{i}$ is dependent on a read operation $r_{i}[y]$ of $T_{i}$ if $r_{i}[y]$ must be scheduled before $w_{i}[x]$.

Definition $18 \mathrm{~A}$ data value $v 1$ is dependent on another data value $v 2$ if the write operation that wrote $v 1$ was dependent on a read operation on $v 2$.

Since the log does not reflect all partial orders among operations in the history, the exact dependencies are hard to compute from the log. However, as per our requirement, the modified log does maintain the order of all conflicting operations. So, it is safe to consider that a write operation of a transaction depends on all read operations of the same transaction that precede the write operation. Assume that $S$ is the set of read operations of a transaction, say 
$T_{i}$, on which a write operation, say $w_{i}[x]$ of $T_{i}$ depends on. But scanning the $\log$, we find that the set of read operations, say $S 1$, that appears before $w_{i}[x]$, is a superset of $S$, i.e., $S \subseteq S 1$. From $S 1$, we would be able to recalculate the value of $x$ although some of the operations in $S 1$ will not be needed in the calculation.

Consider the history $H=r_{1}[a] r_{1}[b] w_{1}[c] r_{2}[a] w_{2}[b] w_{2}[d] c_{2} r_{3}[d] r_{3}[a] c_{1}$ $r_{3}[c] w_{3}[d] c_{3} r_{4}[b] c_{4} w_{5}[a] w_{5}[b] c_{5} r_{6}[b] w_{6}[b] r_{6}[c] w_{6}[c] r_{6}[d] w_{6}[d] r_{6}[a] w_{6}[a]$ $c_{6}$. As per the relaxed definition, $w_{1}[c]$ in history $H$ is assumed to be dependent on $r_{1}[a]$ and $r_{1}[b]$. For that reason, the value of $c$ written by $T_{1}$ is predicted to be dependent on the values of $a$ and $b$, although it may really depend either on $a$ or on $b$.

Notation Let $p=T_{n}\left(q_{1}, q_{2}, \ldots, q_{k}\right)$ denote that the value of $p$ written by $T_{n}$ depends on values of $q_{1}, q_{2}, \ldots$, and $q_{k}$ read by $T_{n}$.

Scanning the history $H$, we get the following dependency of values: $c=$ $T_{1}(a, b), b=T_{2}(a), d=T_{2}(a), d=T_{3}(a, c, d), a=T_{5}(), b=T_{5}(), b=T_{6}(b)$, $c=T_{6}(b, c), d=T_{6}(b, c, d)$, and $a=T_{6}(a, b, c, d)$.

Table 9.1 Data Dependency and Damage Assessment in $H$.

\begin{tabular}{|c|c|c|c|}
\hline$a$ & $b$ & $c$ & $d$ \\
\hline & & $T_{1}(a, b)$ & \\
\hline & $\underline{T_{2}(a)}$ & & $\underline{T_{2}(a)}$ \\
\hline & & & $\underline{T_{3}(a, c, d)}$ \\
\hline$T_{5}()$ & $T_{5}()$ & & \\
\hline & $T_{6}(b)$ & & \\
\hline & & $T_{6}(b, c)$ & \\
\hline & & & $\underline{T_{6}(b, c, d)}$ \\
\hline$\underline{T_{6}(a, b, c, d)}$ & & & \\
\hline
\end{tabular}

Table 1 shows the above data dependencies in $H$ as they occurred over time. Let us assume that $T_{2}$ has been determined as the attacker. Therefore, $b$ and $d$ are concluded to be damaged. Later, when $T_{3}$ writes $d$ after reading it, $d$ continues to be damaged. As the value of $b$ written by $T_{5}$ is independent of any contaminated data, $b$ has been refreshed. $T_{6}$ has read and updated $b$ and $c$ prior to reading the damaged data $d$. Therefore, $b$ and $c$ remain fresh. Likewise, $d$ 
still remains damaged and, furthermore, $a$ is damaged as $w_{6}[a]$ is dependent on $r_{6}[d]$.

Definition 19 A write operation is called a valid write if the value is written by a benign transaction and is independent of any contaminated data.

A valid write on a damaged data refreshes the data. Table 1 also shows how damage has spread and/or refreshed over time. The operations have been underlined to illustrate the damage. Note that the write operation of $T_{6}$ on $a$ may be independent of the value of $d$, and in that case, a will not be contaminated. This dependency can only be determined from the semantics of the updating query that is not taken into consideration in this work. For the simplicity of the recovery process it is safe to assume that $a$ depends on $a, b, c$, and $d$, i.e., $a=T_{6}(a, b, c, d)$.

\subsection{DAMAGE ASSESSMENT}

A graph based approach has been used in this section to observe how damage has spread through the database and which damaged items are refreshed via valid-writes. The graph we use for this purpose is a directed graph. A node in the graph represents a new value of a data item at a given time, and contains information such as the data item name, time of update, and a boolean value indicating whether the data is contaminated. For simplicity, we symbolize each node by either a circle or by a square with the data item name inside it. A circle denotes a clean data item while a corrupted data item is denoted by a square. Each edge represents an update by a transaction that either corrupted the data, or transmitted the damage, or refreshed the data. The nodes for any particular data item are drawn vertically below one another to specify the order among them with respect to the time of update.

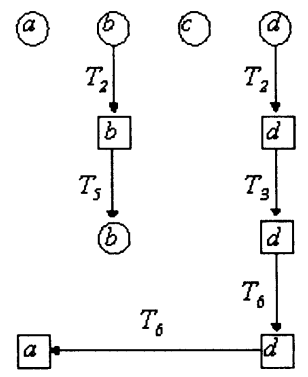

Figure 9.1 Damage Assessment in $H$ using a Directed Graph.

The graph starts with all circular nodes, one for each data item in the database. Whenever an attack is detected, a square-type node is created for each data item that is updated by the attacker. An edge is added to each new node from the initial node that represents the same data item. The edge 
carries the identification of the updating transaction. For each update in the $\log$ that depends on the damaged data, a square-type node is created and an edge is added to the new node from the node(s) on which the update depends. Moreover, whenever a transaction performs a valid-write operation on a damaged data item, a circular node is created for the item and an edge from the previous square-type node of the same item is added showing that the damage has been repaired. Thus, we have three types of edges in the graph: the edges that denote the initial corruption of data by attacking transactions, the edges that show transmission/continuation of damage, and the edges that indicate the refinement of damaged data through valid-writes. Note that no other valid-writes appear in the graph except for those representing the edges of the third type. Figure 1 shows the graph built from history $H$. The graph depicts that items $b$ and $d$ were originally damaged by transaction $T_{2}$ (attacker). Later $b$ has been restored through a valid-write by $T_{5}$. However $T_{3}$ and $T_{6}$ carried on the damage on $d$. Moreover, $T_{6}$ also contaminated $a$ by updating it after reading $d$.

Of all the data dependencies derived from $H$, only the following contribute to the spread and recovery of the damage: $b=T_{2}(a), d=T_{2}(a), d=T_{3}(a, c, d)$, $b=T_{5}(), d=T_{6}(b, c, d)$, and $a=T_{6}(a, b, c, d)$. Also observe that exactly these dependencies are represented by the edges in the graph.

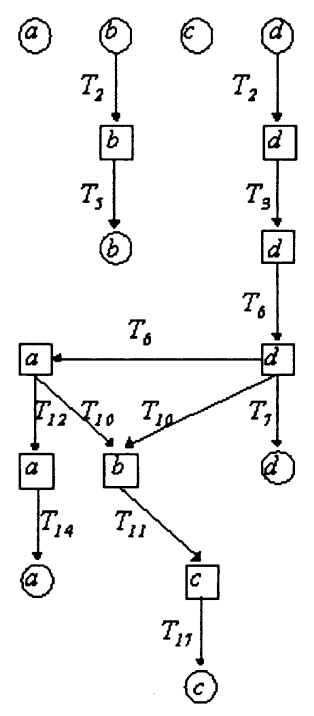

Figure 9.2 A More Complex Graph on Damage Assessment.

Figure 2 displays a more complex graph that is obtained as an extension of the graph in figure 1. These extensions are based upon additional database transactions. Note that at the end, only item $b$ is detected as damaged. During 
recovery, only those transaction operations that contributed for this damage should be recomputed.

\subsection{THE ALGORITHMS}

We present two algorithms here. The first algorithm performs damage assessment and recovery simultaneously; whereas the second algorithm separates damage assessment and recovery processes for improved efficiency. As stated earlier, the modified log stores all read operations of every transaction. This is necessary to determine dependencies of operations. Although the value read can be determined from the after image of the previous write operation on the same data item, for optimization reasons, the value read may as well be stored along with the read operation. In the following algorithm, fresh list and read_list_ $T_{i}$ are lists of records with two fields: data item field and value field. The structure damage item_list includes the list of damaged data as concluded by the algorithm. The value of each damaged item in the damageditem_list that would have been in the database if the attacking transaction had not been executed, is calculated and stored in the fresh list along with its associated data item field. Read_list_ $T_{i}$ contains data items and values read by $T_{i}$.

Notation Let $\left[T_{i}, x, v 1, v 2\right]$ denotes the write operation of $T_{i}$ in the log where $v 1$ and $v 2$ are respectively the before and after images of $x$. The read operations of $T_{i}$ are denoted by $\left[T_{i}, x, v\right]$ which indicates that the value of $x \operatorname{read}$ by $T_{i}$ is $v$.

This notation of representing a write operation of a transaction has been used in [3]. We present our first algorithm on damage assessment and recovery below. This algorithm assesses the damage and calculates the fresh value of the damaged data simultaneously. Note that, as mentioned earlier, the algorithm is based on the assumption that one or more transactions have been identified as attackers. Perhaps the intrusion detection mechanism, or an human analyst checking the values of known data items in the database, or some other means helped in the identification process.

\subsubsection{Algorithm 1}

1. Set damage_item_list $=\{\} ; /^{*}$ Empty set $* /$

2. Set fresh_list $=\{\}$;

3. Scan the log until the end

3.1 For every write operation $\left[T_{i}, x, v 1, v 2\right]$ of an attacker, if $x \notin$ damage_item_list, add $x$ to damage_item_list; and add the record $(x, v 1)$ to fresh_list; /*v1 is the before image of $x^{*} /$

3.2 For any other transaction $T_{j}$ appearing after a write operation of the first attacker, set read_list_ $T_{j}=\{\}$;

3.3 For every read operation $\left[T_{j}, x, v\right]$ of $T_{j}$, add record $(x, v)$ to read_list_ $T_{j}$; 3.3.1 If $x \in$ damage_item_list, replace value $v$ of record $(x, v)$ in read_list_ $T_{j}$ by value of $x$ in fresh_list; 
3.4 For every write operation $\left[T_{j}, x, v 1, v 2\right]$ of $T_{j}$,

3.4.1 If the set of data items in read_list_ $T_{j} \cap$ damage_item_list $\neq \emptyset$, recalculate ${ }^{1}$ new value $v 2$ of $x$, by using values in read_list_ $T_{j}$;

3.4.1.1 If $x \in$ damage_item_list, replace value of $x$ in fresh_list by new value $v 2$;

3.4.1.2 Else

add record $(x, v 2)$ to fresh_list; add $x$ to

damage_item_list;

\subsubsection{Else}

3.4.2.1 If $x \in$ damage_item_list, remove $x$ from damage_item_list; remove the record of $x$ from the fresh_list;

4. For each item in damage_item_list, replace its value in the database by the value in the fresh_list.

Once the list of contaminated data is determined, all data items in the list are blocked from being read by other transactions. This will stop further spread of the damage in the database. However, while recovering the damaged data, overwrites on them by any active transaction must be allowed. Such an overwrite will be a valid-write because no damaged data is allowed to be read. This option will refresh the damaged values. Once a damaged data is refreshed through the recovery process or through a valid-write, the data can be made available for read/write purposes. Next we explain some of the steps in the above algorithm.

Since the attacking transaction may not be the first one of this type, there is a possibility that the data item updated by this particular transaction may have been damaged through a previous attacker. Therefore, in step 3.1, the damage_item_list is checked to see if $x$ is already there. In that case, neither the damage_item_list nor the fresh_list need to be updated. Since the fresh_list should have a correct value of $x$, that value is the before image for this update and must be left there. However, if $x$ was not damaged previously or was damaged but has been refreshed, then the before image of $x$ is the correct value of $x$. The value $v 1$ in item $\left[T_{j}, x, v 1, v 2\right]$ of the log is the before image and, therefore is inserted into the fresh_list. When $T_{j}$ reads a damaged data $x$, in step 3.3.1, the correct value of $x$ that is stored in the fresh_list (see theorem 2 below) is appended to the read_list_ $T_{j}$ for correct calculations of any future updates made by $T_{j}$. In step 3.4.1, $v 2$ is the correct value that should have been in the database if the attacking transaction was not executed. To recalculate the new value of $x$, we need to know the logical operation, say $o$, that was initially used to calculate the after image of $x$. The information on this logical operation, $o$, may not be derived from $v 1$ and $v 2$ only. There are several ways to solve this problem, one of which would be to embed the operation, $o$, in the $\log$, for example $\left[T_{j}, x, o, v 1, v 2\right]$. Step 3.4 .2 .1 will be executed only when the

\footnotetext{
${ }^{1}$ Refer to explanation.
} 
transaction has not read any damaged data but blind-wrote a damaged data. This operation refreshes the damaged value of the data. So the data item is removed from the damage_item_list and from the fresh_list.

9.5.1.1 Proof of Correctness of the Algorithm. The following lemmas and theorems prove that the above algorithm recovers the database to a consistent state that should have been there if the attacking transactions were not executed.

Lemma 1 Every data item that has been updated by an attacker is added to the damage_item_list.

Proof It is obvious from step 3.1 of the algorithm.

Lemma 2 Every data item that (non-transitively) depends on a damaged data written by an attacker is added to the damage_item_list.

Proof Assume that a data item y that directly (non-transitively) depends on $\mathrm{x}$ where $x$ is updated by an attacker $T_{i}$. Now consider the transaction $T_{j}$ that read $x$ and updated $y$. Therefore, the order of $w_{i}[x], r_{j}[x]$, and $w_{j}[y]$ in the history is $w_{i}[x]<r_{j}[x]<w_{j}[y]$, and the operations also appear in the same order in the log. When $\left[T_{i}, x, v 1, v 2\right]$ is found in the log, by lemma $1, x$ is added to damage item_list. Step 3.3 of the algorithm, adds $x$ along with the value read to the read_list_ $T_{j}$ as $\left[T_{j}, x, v\right]$ is detected in the log. Hence, when $\left[T_{j}, y, v 1, v 2\right]$ is discovered, by then $x$ already belongs to both damage_item_list and read_list_ $T_{j}$. Therefore, step 3.4.1. will find that $x \in$ the intersection of these two lists and then step 3.4.1.2 will add $y$ to the damage_item_list.

Theorem 1 A data item $x \in$ damage_item_list iff $x$ is damaged.

Proof If $x$ is damaged by an attacker, by lemma $1 x$ is appended to the list. Similarly, by lemma 2 , if $x$ is damaged after being updated by a nonattacker who read a damaged data written by an attacker, $x$ will be added to the list. There remains a case to discuss when the write operation on $x$ is transitively dependent on a damaged data written by an attacker, but $x \notin$ damage_item_list. In this case, find first such data $x_{1}$, written by $T_{i}$, that is not in the damage_item_list, but the damaged data on which $x_{1}$ depends, say $x_{2}$, is in the damage_item_list. Note that $x_{1}$ may not be different from $x$ itself. Following a proof similar to as in lemma $2,\left[T_{i}, x_{2}, v\right]$ will appear in the log before $\left[T_{i}, x_{1}, v 1, v 2\right]$. Therefore, $x_{2}$ will be in both read_list_ $T_{i}$ and damage_item_list when $\left[T_{i}, x_{1}, v 1, v 2\right]$ is found in the log and thus, $x_{1}$ must have been added to the list at this point as performed by step 3.4.1.2. This proves our above assumption of the existence of such an $x_{1}$ to be false. Therefore, every damaged data $x$ will be added to the damage_item_list. Moreover, $x$ will remain in the list unless $x$ is removed in step 3.4.2.1. This step will execute only when the transaction that wrote $x$, has not read any damaged data. In this case, $x$ is refreshed and no longer remains damaged. 
It remains to prove that no non-damaged item will be in the damage_item_list. Again, assume that $y$ is such an item and was last written by $T_{k}$. Then $T_{k}$ is neither an attacker, nor it had read any damaged data before writing $y$. Therefore, even if $y$ was in damage_item_list, it must have been removed from the list in step 3.4.2.1. This contradiction proves the second part of the theorem.

Lemma 3 If a data item $x \in$ damage_item_list then there is exactly one $(x, v)$ pair in the fresh_list.

Proof For every addition of $x$ to the damage_item_list (in steps 3.1 and 3.4.1.2), there is exactly one $(x, v)$ pair added to the fresh_list. The $(x, v)$ pair is not added to the fresh list anywhere else in the algorithm. Similarly, $x$ removed from the damage_item_list only in step 3.4.2.1, and right there the $(x, v)$ pair is also removed from the fresh list. In no other steps $(x, v)$ pair is removed from the fresh_list. This proves the lemma.

Definition 20 A value $v$ is called the correct value of $x$ if $v$ would have been the value of $x$ in the database in the absence of any attacking transaction.

Theorem 2 The value $v$ in the $(x, v)$ pair in the fresh list is the correct value of $x$.

Proof Considering theorem 1 and lemma 3 together, it is clear that for every damaged item $x$ in the database has exactly one $(x, v)$ pair in the fresh_list. It remains to prove that $v$ is the correct value of $x$. This is proved by induction as follows.

Assume that all the data items in the damage_item_list are the result of updates of the first transaction that did the damage. Obviously, the transaction is the attacker. As per lemma 3, each of these damaged data also appear in the fresh list. As per step 3.1 of the algorithm, for every $(x, v)$ pair, $v$ is the before image of $x$, and this before image is the after image of a valid write by the last transaction that wrote $x$ before the attacker. This value should have been in the database if the attacking transaction was not executed, i.e., the fresh_list starts with all correct values in all (data item, value) pairs.

Next, we intend to show that for every addition of $(x, v)$ to fresh list, $v$ is the correct value of $x$. There are two cases where such a pair can be added to the fresh_list: when a non attacking transaction spreads the damage, and when another attacking transaction executes. Case 1 occurs if a transaction, $T_{i}$, reads a damaged data $x$ before writing any data $y$ ( $x$ and $y$ may be the same). In step 3.3 .1 of the algorithm, whenever $T_{i}$ reads a damaged data $x$, the value read is ignored and the value $v$ of $x$ in the fresh list is inserted into the read_list_ $T_{i}$. This value is the correct value of $x$ since the fresh_list, as proved in the previous paragraph, already contains correct value of $x$. When $T_{i}$ writes $y$, the new value of $y$ is calculated in step 3.4.1 from the read_list_ $T_{i}$ which contains only correct values. Thus, the new value of $y$ is a result of a valid write and is correct. This value is replaced in step 3.4.1.1 (if $y$ is already in 
fresh_list), or inserted into the list in step 3.4.1.2 (if $y$ is not in the fresh_list). In case 2 , another attacking transaction $T_{i}$ updates $x$ and therefore $(x, v)$ is inserted into fresh_list. There are two possibilities here too. First, $x$ may have been damaged by another transaction and hence $(x, v)$ pair is already in the fresh list. In this case, the fresh list is not updated and so continues to have the correct value of $x$. Secondly, $x$ may not have been damaged before. Therefore, as explained in the previous paragraph, the before image of $x$ after $T_{i}$ updates $x$ is the correct value of $x$ and is inserted into the fresh_list. This completes the proof.

Theorem 3 The database state produced by this algorithm is the same as the state that would have been produced if there was no attack on the database.

Proof It is clear from theorem 1, lemma 3, and theorem 2 that every damaged data in the database has its correct value in the fresh_list. Since, step 4 of the algorithm replaces the value of every damaged data in the database by the value of the same in the fresh_list, the database will not have any effect of attacking transactions at all. Moreover, the data items that were either not affected by the attackers or were refreshed later on through blind-writes will have their correct values in the database and will not be modified during the recovery process. Therefore, none of the effects of any good transactions will be lost.

Although the previous algorithm will precisely detect all damaged data items and repair them, it will block all active transactions in the system until the recovery process is complete. During this process, a significant delay is expected due to the computation required to determine the valid values of all damaged data and also for the disk accesses needed to the log that keeps a copy of all committed transactions in the system. The next algorithm solves this problem by first determining the set of damaged data and then making non-damaged data available to active transactions in the system. This will make the unaffected part of the database operative while the recovery continues. As pointed out earlier, during the recovery process the damaged data are available for blind-writes. This further increases the availability of data in the system while the recovery is in progress.

It is possible to accomplish the above-mentioned goal by removing the fresh_list and the related calculation from the previous algorithm during the damage assessment phase. Then, during recovery, the new value of each damaged data can be calculated. In order to do this, however, the partial order of all transactions that have accessed damaged data is needed. The process involves a second reading of the log incurring more disk accesses. To resolve this problem, we use a different data structure, damage_audit_table, as described next.

The auxiliary structure damage_audit_table stores information about transactions that have either corrupted the data, or transmitted/continued the damage, or refreshed the data. Transaction records are appended to the table in the same order as they appear in the log and each record has four fields: transaction_id, data_written, valid_read, and invalid_read. The data_written field 
stores only data items with values that are either written by an attacker, or data that are dependent on the damaged data, or the fresh value of a previously damaged data after a valid-write. Notice that all data items in this column also appear as nodes in the damage assessment graph and vice versa. While the valid_read field stores all non_damaged data with their values as read, the invalid_read field keeps the damaged data along with their values that are read by the transaction. The algorithm consists of two phases: a damage assessment phase and a recovery phase. We present the damage assessment phase next. Again, note that the following algorithm requires the identification of the attacking transaction(s) as assumed for the previous algorithm.

\subsubsection{Algorithm 2.1 (Damage Assessment)}

1. Initialize damage_audit_table $=\{\} ;$ and damage_item_list $=\{\}$;

2. Scan the log until the end

2.1 When an attacker, $T i$, is found, add a new record with the transaction_id of $T_{i}$ into damage_audit_table;

2.1.1 For every write operation $\left[T_{i}, x, v 1, v 2\right]$ in the $\log$, add $(x, v 1)$ order pair to data_written column of $T_{i}$ 's record; and add $x$ to the damage_item_list if it is not there;

2.2 For any other updating transaction $T_{j}$ appearing after a write operation of the first attacker, add a record for $T_{j}$ into damage_audit_table;

2.2.1 For every read operation $\left[T_{j}, x, v\right]$

If $x \in$ damage_item_list, add $x$ to invalid_read column of $T_{j}$;

Else add $(x, v)$ pair to valid_read column of $T_{j}$;

2.2.2 For every write operation $\left[T_{j}, x, v 1, v 2\right]$

If invalid_read column of $T_{j}$ is $\neq \emptyset, /^{*} T_{j}$ has spread the damage*/ add $(x, v 2)$ pair to data_written column of $T_{j}$; and add $x$ to the damage_item_list if it is not there;

Else

If $x \in$ damage_item_list, / $T_{j}$ has a valid-write on damaged data */

write $(x, v 2)$ pair to data_written column of $T_{j}$; and remove $x$ from damage_item_list;

2.2.3 If [Commit, $T_{j}$ ] found,

If both invalid_read and data_written columns of $T_{j}$ are empty, remove $T_{j}$ 's record from damage_audit_table

Else If [Abort, $T_{j}$ ] found, remove $T_{j}$ 's record from damage_audit_table.

Once the damage list is determined, all non-damaged data are made accessible to users while the recovery process continues. Moreover, users are allowed to make blind-updates on damaged data. Next, we present the recovery phase 
of the algorithm. This algorithm uses the damage_audit_table and the damage item_list as input in determining the correct values of the damaged data.

\subsubsection{Algorithm 2.2 (Recovery)}

1. Scan records in damage_audit_table until the end;

1.1. For every attacking transaction other than the first one,

1.1.1 For each $(x, v)$ pair in data_written column, substitute $v$ by the before image of $x$;

/* For the first attacker, the before image is already there */

$/^{*}$ If multiple attackers have written $x$ consecutively, the before image of $x$ for each of these transactions is that of the first transaction */

1.2. For every non-attacking transaction with non-empty invalid_read column

/* These transactions have spread damage */

$/^{*}$ Any non-attacking transaction with an empty invalid_read column in damage_audit table, has refreshed some data items and their records need not be modified */

1.2.1 For every $x$ in invalid_read column, scan the data_written column upward starting from the previous record in damage_audit table to find the first $(x, v)$ pair; $/ *$ the last update on $x^{*} /$ add $(x, v)$ pair into the valid_read column; and remove $x$ from invalid_read column;

1.2.2 For every $x$ in data_written column, calculate the value $v$ of $x$ using values in the valid_read column; and substitute $(x, v)$ in data_written column; $/^{*} v$ is the correct value of $x * /$

2. For every $x$ in damage_item_list, check the new log that has just been created while the recovery process was in progress;

2.1 If $x$ is not modified in the $\log , /^{*}$ otherwise, the update on $x$ is a valid-write */

scan data_written column of damage_audit_table from bottom-up to find first $(x, v)$ pair, and substitute the value of $x$ in database with $v$.

\subsection{CONCLUSIONS}

The existing recovery algorithms are not designed to operate in an information warfare environment. This research offers recovery algorithms that restore the database to a consistent state by recomputing the affected operations of all benign transactions that follow the attacker. For this purpose, the transaction $\log$ is modified to store the read operations of all transactions in addition to their write operations. The first algorithm performs the damage assessment and recovery simultaneously while these two methods are separated in the second algorithm. The first algorithm requires that the entire system is brought to a halt until the recovery is complete. The second algorithm, which comprises of two phases, releases the unaffected part of the database soon after the damage 
assessment phase is completed. This makes the system available to users while the recovery process continues.

\section{Acknowledgments}

1. This work was supported, in part, by the US AFOSR Summer Faculty Research Program.

2. The authors would like to thank Prof. Sushil Jajodia for his valuable suggestions on this research.

\section{References}

[1] P. Ammann, S. Jajodia, C. McCollum, and B. Blaustein. Surviving Information Warfare Attacks on Databases. In Proc. IEEE Symposium on Security and Privacy, pages 164-174, Oakland, California, 1997.

[2] P. Bernstein, V. Hadzilacos, and N. Goodman. Concurrency Control and Recovery in Database Systems. Reading, Massachusetts: Addison-Wesley, 1987.

[3] R. Elmasri and S. Navathe. Fundamentals of Database Systems. Menlo Park, California: Addison-Wesley, 2nd edition, 1994.

[4] R. Graubart, L. Schlipper, and C. McCollum. Defending Database Management Systems Against Information Warfare Attacks. Technical report, The MITRE Corporation, 1996.

[5] J. Gray and A. Reuter. Transaction Processing: Concepts and Techniques. San Mateo, California: Morgan Kaufmann, 1993.

[6] H. Korth and A. Silberschatz. Database System Concepts. New York : McGraw-Hill, 2nd edition, 1991.

[7] T. Lunt. Using Statistics to Track Intruders. In Proceedings of the Joint Statistical Meetings of the American Statistical Association, 1990.

[8] T. Lunt. A Survey of Intrusion Detection Techniques. Computers \& Security, 12(4):405-418, 1993.

[9] T. Lunt and et. al. Knowledge-based Intrusion Detection. In Proceedings of the 1989 AI Systems in Government Conference, 1989.

[10] J. McDermott and D. Goldschlag. Storage Jamming. In D. Spooner, S. Demurjian, and J. Dobson, editors, Database Security IX: Status and Prospects, pages 365-381. London: Chapman \& Hall, 1996.

[11] J. McDermott and D. Goldschlag. Towards a Model of Storage Jamming. In D. Spooner, S. Demurjian, and J. Dobson, editors, Proc. of the IEEE Computer Security Foundations Workshop, pages 176-185, Kenmare, Ireland, 1996. 